[18] Shalimova, S. O. (Ed.) (2012). Rak v Ukrayini, 2010-2011. Zahvoryuvanist, smertnist, pokazniki diyainosti onkologichnoiy sluzhbi. Biuleten natsionalnogo kantser-reestru Ukraiyini. Kyiv, 13, 51.

[19] Davydov, M. I., Gantsev, Sh. Kh. (2013). Onkologiya. Moscow: GEOTAR-Media, 920.

[20] Ionova, T. I., Novik, A. A., Suhonos, Yu. A. (1998). Kachestvo zhizni onkologicheskih bolnyih. Voprosi onkologii, 44 (6), 749-752.

[21] Novik, A. A., Ionova, T. I., Kaynd, P. (1999). Kontseptsiya issledovaniya kachestva zhizni v meditsine. Saint Petersburg: Elbi, 140.

\title{
THE EFFICIENCY OF GLUCOCORTICOID THERAPY IN SECONDARY-PROGRESSIVE COURSE OF MULTIPLE SCLEROSIS
}

\author{
Vitaliy Vasylovskyy \\ Department of Neuroinfection and Multiple Sclerosis \\ State institution "Institute of Neurology, Psychiatrist and Narcology of the National Academy of \\ Medical Sciences of Ukraine" \\ 46 Akademika Pavlova str., Kharkiv, Ukraine, 61068 \\ vvasylovskyy72@gmail.com
}

\begin{abstract}
We have investigated the efficacy of pulse-therapy with glucocorticoids (GC) at different time stages (in debuts, at the recurrent stage and at the stage of progression) of secondary progressive course (SPC) of multiple sclerosis (MS) in 70 patients ( 57 women and $13 \mathrm{men}$ ) at the ages from 28 to 67 years (mean age $45 \pm 2.5$ years). The duration of the disease accounted for 7 up to 34 years (average duration was $19.8 \pm 2.3$ years). We have conducted 438 courses of GC therapy: at the onsets - 11, at the recurrent stage (RS) -178 and at the stage of secondary progression-249.

The efficacy of hormonal therapy was assessed taking into account the following criteria: the dynamics of regression of neurological symptoms under the influence of the first course of GC therapy at the stage of onsets; a comparative evaluation of remission's duration after admission and without taking GC at the onsets; duration of RS depending on the duration of remissions after the first course of GC therapy; a comparative evaluation of remissions' duration after the 1st (at the stage of onset and/or on the RS), and the period of stabilization on the SPS before the last courses of GC; the variants of secondary progression under the influence of GC courses; scores according the EDSS disability scale after the 1st and before the last course of GC therapy; the rate of progression under the influence of the repeated courses of GC therapy.

We have defined the three categories of efficacy at the repeated courses of pulse therapy with GC: the moderate efficacy, the low efficacy, the lack of efficacy. We have not observed the high efficacy in patients with SPC.

The patients with MFR $<1.0$, among which the pulse therapy with GC promoted the prolongation of RS, relieved the severe (less often) and moderate (more often) relapses, the outcome of which was accompanied by a moderate and stable neurologic deficit, were subsumed under the subgroup with moderate efficacy (21 individuals). The most favorable progressive variant of progression prevailed in these patients after transformation of RS into SPS.

The patients with different rate of MFR ( 9 - with MFR $<1.0$ and 12 - with MFR $>1.0$ ), with short (more often) and moderate (less often) RS, during which the accumulation of neurological deficit due to the frequent and heavy relapses had occurred, were subsumed under the subgroup with low efficiency (21 individuals). After transformation into SPS, the recurrent variant of progression prevailed in these patients.

The patients who were characterized by short RS, by predominance of severe and prolonged relapses, the MFR value greater than 1.0, the steady accumulation of a pronounced and persistent neurologic deficit, a high rate of progression and high scores on the EDSS disability scale more than 6.5 points) were subsumed under the subgroup with the lack of efficacy (28 individuals). After transformation in the SPC, the most unfavorable variant of progression prevailed (21 patients); significantly less frequent were the recurrent ( 5 patients) and a combination of a steady and recurrent ( 2 patients) progression. The persistent lack of efficacy of the hormonal therapy in this subgroup of patients was most likely associated with the genetically determined low individual sensitivity to GC.
\end{abstract}


Therefore, the results of our study showed that the efficacy of GC therapy in SPC of MS is determined by the complex interaction of clinical indicators having the prognostic value, as well as by the number of the genetic factors, which require their further observation.

Keywords: multiple sclerosis, secondary-progressive course, pulse-therapy with glucocorticoids, treatment effectiveness.

\section{Introduction}

The modern algorithm for the treatment of multiple sclerosis (MS) is based on "four whales": relief of exacerbations with the help of glucocorticoids (GC); immunotherapy with drugs that change the course of MS (PITRS), symptomatic therapy, which removes various clinical manifestations of the disease and a complex of rehabilitation measures, including the development of adaptive strategies for reducing disability $[1,2]$.

As first-line drugs that relieve exacerbations of MS, are GC (prednisolone, methylprednisolone), the clinical effect of which is due to immunosuppressive and anti-inflammatory action. GC reduce edema in the inflammatory focus, inhibit the production of pro-inflammatory cytokines, express the main histocompatibility complex (HC) of class II and adhesion molecules, stabilize the permeability of the blood-brain barrier (BBB), induce apoptosis of activated lymphocytes [3].

Hormone therapy in MS is important not only as a factor that suppresses the autoimmune process, but also as a form of replacement therapy in connection with the development of glucocorticoid insufficiency in this disease, which changes immunological reactivity in the direction of enhancing allergic manifestations and contributes to the demyelination process [4].

As it known, the secondary progressive course (SPC), in contrast to the recurrent course $(\mathrm{RC})$, is characterized by a more unfavorable course due to the progression of the process leading to accumulation of a neurological deficit and persistent disability $[5,6]$. Therefore, timely and adequate appointment of GC in the early stages of the disease, i.e. in debuts and on the recurrent stage (RS), can postpone further progression of the process [7].

The revealed effects of GC allowed us to reconsider traditionally a skeptical attitude toward the appointment of GC in progressive type of flow (PTF), for which the spectrum of therapeutic measures until recently was forcedly limited by the cytostatics, that give a significant number of complications during long-term admission $[8,9]$.

As for the SPC, in this form it is proved that degenerative-axonal lesions are combined with autoimmune inflammatory changes of varying severity [10].

Consequently, the activity of the demyelinating process at the stage of secondary progression (SSP) is comparable with the activity of relapses in RC, despite a different time algorithm for the development of the inflammatory process and significant differences between these types of flow, manifested by clinical-immunological and clinical-morphological dissociations, named $\mathrm{Ch}$. Pozer as "phenomenon of the iceberg" [11]. This provision is the evidence for the use of active immunosuppressive therapy of GC for this category of patients [12].

In recent decades, a highly effective pulse-therapy with methylprednisolone has been used to relief relapses, which has a significant advantage over prednisolone due to the presence of a methyl group that can penetrate the cell membrane and bind to intracellular receptors [13]. Due to the powerful anti-inflammatory and anti-edematous effects, the proposed scheme (intravenous drip high-dose up to $1000 \mathrm{mg}$ of metipred or solu-medrol for 5-7 days) remains effective for 1.5 months after the development of exacerbations in the form of a significant reduction in brain volume, recovery affected by the demyelinating process of the functions of the central nervous system, the normalization of the permeability of the BBB. In addition, GC can prevent the development of brain atrophy, preventing early formation of persistent disability, by slowing the accumulation of residual neurological deficits [14].

\section{Aim of the research}

The aim is to evaluate the effectiveness of hormonal pulse therapy at different time stages of the secondary progressive course of multiple sclerosis. 


\section{Materials and methods}

In the study, which was conducted in the Institute of Neurology of Psychiatry and Narcology of the National Academy of Medical Sciences of Ukraine (GU INPN NAMN), Department of Neuroinfections and Multiple Sclerosis, from 2008 to 2017, The patients with SPC MS were selected and monitored. GC treatment was performed at different time stages of the SPC (in the beginning, at the recurrent stage (RS) and at the stage of secondary progression (SSP)) in 70 patients (57 women and 13 men) aged from 28 to 67 years (mean age $45 \pm 2.5$ ) years) with the duration of the disease from 7 to 34 years (mean duration - (19.8 \pm 2.3$)$ years).

In the research next methods were used: clinical-neurological, statistical.

The following clinical indicators were taken into account in characterizing the course of the clinical course of SPC MS: the age of the manifestation of clinical symptoms, the severity of the debuts, the duration of remission after the debut and the duration of the RS, the severity and number of relapses in the RS, the mean frequency of relapses in the RS, the duration of the SSP, the progression variants, the rate of progression, the degree of disability on the EDSS scale.

The total number of courses of GC therapy in 70 patients, including all stages of the disease, was 438, including 11 at the debut stage, 178 at the RS, and 249 at the EVP.

The effectiveness of hormonal therapy was evaluated taking into account the following criteria:

- dynamics of regression of neurological symptoms under the influence of the first course of GC therapy at the stage of debuts;

- comparative evaluation of the duration of remission after admission and without taking $\mathrm{GC}$ in the beginning;

- the average frequency of relapses against the background of repeated courses of GC therapy in the RS;

- the duration of the RS depending on the duration of remission after the first course of GC therapy;

- comparative evaluation of the duration of remissions after the first (at the stage of debut and/or on the ER RS and the period of stabilization on the SSP before the last course of the GC;

- variants of secondary progression under the influence of GC courses;

- scores on the EDSS disability score scale after the 1st and before the last course of GC therapy;

- the rate of progression (the sum of the EDSS disability score difference between the first and before the last GC course for each patient in relation to the total number of patients in each group) under the influence of repeated courses of GC therapy.

Statistical processing of the results of the study was carried out using a package of statistical programs "Statgraph". It was determined $\mathrm{n}$ - the number of patients; $\mathrm{M}$ is the average value of the indicator; $\mathrm{m}$ is the standard deviation of the indicator.

\section{Results}

The age of the debut at the time of evaluation of the effectiveness of treatment with GC varied significantly (from 14 to 44 years) and averaged $(26.1 \pm 2.0)$ years.

Comparative evaluation of the severity of the debuts revealed a significant predominance of debuts of moderate severity compared with light and heavy debuts. In turn, heavy debuts were significantly less frequent than light debuts (Table 1).

Table 1

The frequency of debuts of varying severity with SPC MS, M $\pm \mathrm{m}, \%$

\begin{tabular}{cc}
\hline Severity of the debuts & SPC $(\mathbf{n}=\mathbf{7 0})$ \\
\hline Light debuts & $27,5 \pm 7,4^{1,2)}$ \\
Medium debuts & $62,5 \pm 8,1^{1)}$ \\
Heavy debuts & $10,0 \pm 5,1^{1,2)}$
\end{tabular}

Note: $n$ is the number of patients; ${ }^{1)}-p<0.05$ - between medium-heavy debuts and debuts of light and heavy severity; ${ }^{2)}-p<0.05-$ between light and heavy debuts 
Pulse therapy of GC with the manifestation of clinical symptoms in the debuts received only $11(15.7 \%)$ patients who had been diagnosed with MS in a timely manner. The experience of GC in the debuts, despite the low percentage of patients, proved to be highly effective in patients with a medium severity of debuts (8 people) who had a full regress of neurologic symptoms with the release of clinical remission. In patients with heavy debuts ( 3 people), the output from the debuts was protracted, accompanied by a minimal regression of the neurological deficit with the outcome of incomplete clinical remission.

The average duration of remission after the debut in all patients with SPC, as well as for subgroups of patients taking and not taking GC, did not reveal significant differences. However, in patients with medium debut with GC, remission after debut was significantly longer than in patients with heavy debuts (5.3 \pm 1.4 and $3.2 \pm 0.6$ years, respectively) (Table 2).

\section{Table 2}

The duration of remission after debut under the influence of the first course of GC therapy in patients with $\mathrm{SPC} \mathrm{MS}, \mathrm{M} \pm \mathrm{m}$, in years

\begin{tabular}{cc}
\hline Group of patients & The duration of remission after debut $(\mathbf{n}=\mathbf{7 0})$ \\
\hline In the general group of patients & $3.2 \pm 0.6$ \\
In the group after taking GC & $2.8 \pm 1.2$ \\
In the group without GC & $3.1 \pm 0.6$
\end{tabular}

Note: $n$ is the number of patients

Features of the course of RS, primarily its duration, frequency and severity of relapses, as well as the rate of accumulation of a neurological deficit, play a key role in starting the process of transforming RS into SSP. To implement this process, a complex selective structural reorganization of clinical indicators in the RS is necessary, the leading role among which belongs to the severity of relapses. The increase in severe relapses during the course of the RS flow accelerates the transformation of RC into the SPC $[15,16]$.

In terms of duration, the average duration of the RS was $10.4 \pm 3.9$ years and was divided into short (from 2 to 5 years), moderate (from 5 to 8 years) and long (more than 8 years).

Neurological symptoms during exacerbations in the RS affected the leading functional systems with the predominance of pyramidal and cerebellar syndromes. As a rule, relapses of different severity (light, medium and heavy) alternated among themselves in the vast majority of patients as the RS progressed. Mild relapses were characterized by rapid rates of clinical symptomatology, short duration (no more than 3-4 weeks), mono- or oligo-syndromic symptoms with minimal signs of rapidly regressing neurological deficit. With relapses of moderate severity, oligo- or polysyndromic symptoms prevailed, predominantly developing at a gradual pace, the formation of a moderate neurological deficit with a slower rate of regression (up to 2 or more months) with an incomplete clinical remission. For severe relapses, the slow pace of formation of severe polysyndrome symptomatology with subsequent partial and differentiated for different functional systems regress and delayed output (for 3 or more months) in short and incomplete clinical remissions were characteristic.

The total number of relapses in the RS in 70 patients was 366 . The mean frequency of relapses (MFR - the ratio of the number of relapses to the duration of the RS) is $0.9 \pm 0.2$. However, this indicator was not sufficiently informative, since the range of fluctuations for each patient was from 0.1 to 2.8 .

The analysis showed that in all patients a low MFR value $(<1.0)$ indicated a rare recurrence and prolonged RS; an increase in MFR $(>1.0)$ occurred with frequent recurrences and short duration of the RS (Table 3).

This position is confirmed by the data obtained in the analysis of the average duration of the RS, depending on the size of the MFR, which was significantly higher in the case of an MFR $<1.0$ than with an MFR value $>1.0$ (Table 4). Consequently, an increase in the MFR as an integral 
measure of the relationship between the duration of the RS and the number of relapses may serve as one of the probable criteria for the nearest transformation of the RS into the SSP.

Table 3

The average frequency of recurrences in the RS with SPC MS on the background of repeated courses of GC, $\mathrm{M} \pm \mathrm{m}$, in years

\begin{tabular}{cc}
\hline Average meaning of MFR & SPC $(\mathbf{n}=\mathbf{7 0})$ \\
\hline MFR of all patients. including: & $0.9 \pm 0.2$ \\
MFR $<1.0$ & $0.5 \pm 0.1^{1)}$ \\
MFR $>1.0$ & $1.6 \pm 0.7^{1)}$
\end{tabular}

Note: $n$ - the number of patients; ${ }^{\text {I) }}-p<0.05$ - differences between $M F R<1.0$ and MFR $>1.0$

Table 4

Duration of the RS, taking into account the mean frequency of relapses (MFR) in case of SPC MS, M \pm m, in years

\begin{tabular}{cc}
\hline Duration of the RS & SPC $(\mathbf{n}=\mathbf{7 0})$ \\
\hline Duration of the RS at MFR $<1.0$ & $14.7 \pm 2.1^{1)}$ \\
Duration of the RS at MFR $>1.0$ & $3.9 \pm 0.9^{1)}$
\end{tabular}

Note: $n$-the number of patients; ${ }^{1)}-p<0.05$-differences between $M F R<1.0$ and MFR $>1.0$

Analysis of the frequency of relapses of varying severity in all patients with SPC showed a significant predominance of relapses of moderate severity over light and severe relapses that occurred almost at the same frequency (Tab. 5).

Table 5

Severity of relapses in the RS with SPC MS on the background of repeated courses of GC, M \pm m, \%

\begin{tabular}{|c|c|}
\hline Severity of relapses in the RS & SPC $(\mathbf{n}=\mathbf{7 0})$ \\
\hline Light & $27.2 \pm 7.5^{1)}$ \\
Medium & $51.9 \pm 8.5^{1)}$ \\
Heavy & $20.9 \pm 6.7^{1)}$
\end{tabular}

Note: $n$-the number of patients; ${ }^{1)}-p<0.05$ - between relapses of medium severity and relapses of light and heavy severity

The first course of GC therapy was given to 59 out of 70 patients with SPC. The total number of courses was 178, including 104 for relapses of moderate severity, 74 for severe recurrences. With light relapses, GC therapy was not used, despite the fact that a significant part of patients of this subgroup had radiological activity, according to MRI. Such a violation of the protocol providing for the appointment of hormonal therapy for all relapses, regardless of their severity, led to a further increase and increase in relapse, the accumulation of a residual neurological deficit, a reduction in the duration of RS, an increased risk of transformation of RS into secondary progression.

The clinical effect under the influence of courses of GC therapy was characterized by a differentiated regression of the neurological deficit. This allowed us to distinguish conditionally "well-managed" and "poorly managed" symptoms in each functional system. In the pyramidal syndrome, reversal of the spinal tone was primarily affected by spasms, whereas the restoration of strength in the legs depended on the severity of the paresis. Within the cerebellar-atactic syndrome, a reduction in the amplitude of horizontal nystagmus and shakiness in walking, as well as an improvement in the finger-nasal test, are among the "well-controlled" symptoms. Significantly less frequent reversal was the execution of the knee-heel test and static ataxia in the Romberg sample. 
The regression of sensitive disorders took place differentially and depended on their nature. The most "controlled symptoms" were violations of pain sensitivity and astereognosis, while normalization of proprioceptive and temperature sensitivity occurred slowly and, as a rule, partially. In stem disorders, vestibular syndrome, vertical nystagmus, impairment of the function of the facial musculature, as a consequence of facial nerve lesions, were more often subjected to significant regression; rarer - a variety of oculomotor disorders. Sphincter disorders, depending on the degree of their decompensation, were usually partially regressed with significant individual differences.

Thus, the analysis of the effectiveness of pulse therapy of GC indicates that the RS with relapses of varying severity formed a "dissociation syndrome" with selective and differentiated regression of clinical symptoms in individual functional systems.

Remission after the first course of GC therapy was divided into short (up to 1 year), moderate (1 to 2 years) and prolonged (more than 2 years), but their frequency did not have significant differences (short - 31.6 \pm 9.6 , moderate $-38.8 \pm 9.7$, long-term $-29.6 \pm 8.8$ ).

A directly proportional relationship was established between the mean duration of remission (MDR) after the first course of GC therapy (2.3 \pm 0.7$)$ years and the duration of the RS. Thus, with prolonged RS, MDR was significantly higher than for its short and moderate duration (Table 6).

Thus, prolonged remissions after the first course of GC contributed to the prolongation of the RS. Consequently, this indicator can act as a prognostic marker, indicating a reduction in the risk of transformation of RC into the SPC. This provision is of great practical importance for the timely and adequate development of a treatment strategy, including modifying agents and cytostatics, which in the RS will create real prerequisites for preventing further rapid progression of the disease.

\section{Table 6}

Dependence of the average duration of remission after the first course of GC therapy on the duration of the $\mathrm{RS}$ in cases of SPC MS, in years

\begin{tabular}{cc}
\hline Duration of RS & Average duration of remission \\
\hline short (2 to5 years) & 0.5 \\
moderate (5 to5 8 years) & 0.7 \\
prolonged (more than 8 years) & 1.8
\end{tabular}

The different sensitivity to repeated courses of hormonal therapy, obtained during the analysis of clinical indicators at the RS, led to the fact that the process of transformation in SSP proceeded at different rates in different patients.

Clinical analysis of the course of SSP made it possible to distinguish three main variants of progression, evidencing its complex structural and functional organization:

- steady variant, current without clinically outlined periods of stabilization;

- recurrent variant, proceeding in the form of abrupt deteriorations, reminiscent of relapses, at the exit from which periods of stabilization of different duration were observed;

- progressive variant, which is an alternation of periods of slow progression of neurological symptoms, alternating with periods of stabilization of different duration.

For the first two variants (steady and relapsing), usually unfavorable, the development of coarse and persistent polysyndrome neurologic symptoms, absence or relative rarity of dissociation syndromes, high rate of progression is characteristic. As a result, a deep degree of disability and persistent therapeutic resistance to GC therapy are formed. Progressive version of progression is more favorable and is characterized by the absence of a severe neurological deficit, a longer period of residual ability to work, better socio-psychological adaptation, and greater effectiveness of pathogenetic therapy. The character of the further progression was determined not only by the variants, but also by the rate of growth of the neurological symptomatology - rapid, moderate and delayed. At a rapid pace, the steady and recurrent options prevail or alternate; at moderate and especially slow rates - a progressive variant. 
At the time of evaluating the results of treatment with GC, the average duration of the SSP was (7.2 \pm 1.4$)$ years. At this stage, 249 courses of GC therapy (3.5 - the average number of courses per patient) were conducted. The need for more frequent courses on SSP, compared with the RS, indicates a steady decline in the effectiveness of hormone therapy after the formation of progressive flow.

However, against the background of repeated courses of GC, the structural characteristic of the SSP was clearly individual. A clear relationship was found between the magnitude of the MFR on the RS and the variants of secondary progression. For example, with $M F R<1.0$, the most favorable - a progressive variant of progression was observed in 21 patients, and a recurrent variant was observed in 14 patients. With an MFR $>1.0$, the most unfavorable variant prevailed, which was observed in 26 patients, while the recurrent variant was found in only 9 patients.

One of the important criteria for the effectiveness of GC treatment at the end of the study was a comparative assessment of the duration of remission in the RS after the first and the period of stabilization before the last course of GC. According to the data obtained, the duration of stabilization before the last course was significantly shorter in patients with SSP than the remission of the RS after the first course of GC (Table 7).

The received data confirm the position that with prolonged use of GC therapy causes oppression of the hypothalamic-pituitary-adrenal system, contributes to the formation of steroid dependence, which leads to stable hormone-dependent forms and further progression of the disease.

As one of the leading criteria for the effectiveness of hormonal therapy in the SSP, a comparative assessment of the EDSS scores on the EDSS disability score was made after the first and before the last course of pulse therapy for GC.

\section{Table 7}

The duration of remission on the SP after the first and stabilization before the last course of GC in patients with SPC MS, M $\pm m$, in years

\begin{tabular}{cc}
\hline Index & SPC (n=70) \\
\hline The duration of remission on the SP after the first course of GC & $2.3 \pm 0.7^{1)}$ \\
The duration of stabilization before the last course of GC & $1.2 \pm 0.2^{1)}$ \\
Note: $n$ - the number of patients; ${ }^{l)}-p<0.05$ - between the first and before the last course of the GC
\end{tabular}

The average meaning on the EDSS disability score after the first course of GC therapy were (3.2 \pm 0.5$)$ points. Analysis of the gradual course of the disease, despite repeated courses of hormonal therapy, indicates that on the SSP before the last course of GC therapy in the vast majority of patients, the value of the mean scores on the EDSS disability scale increased to $6.1 \pm 0.9(p<0.05)$.

The negative dynamics of scores on the EDSS scale corresponded to the integral index, as the rate of progression, which by the end of the study were 2.3 .

\section{Discussion}

At the present time, according to different authors, the question of the efficacy of GC in patients with SPC MS [17, 18] remains controversial. The prognosis of the transformation of the $\mathrm{RS}$ in the SPC with the timely hormonal therapy of exacerbations decreases according to the data of the authors [19]. The prognosis of the course of SPC in the course of GC is unclear. However, there is no correlation between the increase in neurological deficit in patients with SPC MS in the use of GC [20].

In this study, an integrative assessment of the effect of repeated courses of pulse therapy of GC, performed at different time stages with SPC MS, made it possible to distinguish three subgroups of patients - with moderate and low efficacy, as well as lack of efficacy. High efficiency, which assumes no risk of transforming the RS into an SPC, with this type of flow absent.

Moderate efficacy was detected in 21 patients with an MFR $<1.0$. This category of effectiveness included patients who, under the influence of GC treatment, were able to manage severe (less often) and moderate (more often) relapses, with a moderate and stable neurological deficit. After 
the transformation of RS into an SPC, the most favorable progressive variant of progression was characteristic for these patients.

Low efficacy was also obtained in 21 patients, including 9 people with $M F R<1.0$ and in 12 people with MFR $>1.0$. For this subgroup of patients, in spite of the different size of the MFR, a short (more often) and a moderate (less frequently) RS appeared to be characteristic, during which a general tendency was observed to increase and increase relapses with the accumulation of a neurological deficit. As a result, there was a transformation in SSP, in which a recurring variant of progression prevailed.

Lack of efficacy was obtained in 28 patients who had short RS, predominance of severe and prolonged relapses, greater than 1.0 MFR, a steady accumulation of a pronounced and persistent neurologic deficit, a high rate of progression and high scores on the EDSS disability score (more than 6.5). After transformation in SSP, the most unfavorable variant of progression prevailed (21 patients); significantly less frequent recurrent (5 patients) and a combination of a steady and recurrent ( 2 patients) progression.

\section{Conclusions}

1. Under the influence of repeated courses of GC therapy with SPC MS there is a complex systemic reorganization of the clinical indices characterizing the time stages of the course of the disease. Clinical analysis of the dynamics of various indicators allowed to develop criteria for the effectiveness of treatment for patients with SPC MS.

2. Allocation of subgroups of patients with only moderate and low efficacy did not exclude the risk of transformation into the SPC. However, this process occurred at different times and was accompanied by significant individual differences.

3. Patients with moderate efficacy of hormonal therapy were characterized by an increase in the duration of remission after debut, a decrease in the mean frequency of relapses $(\mathrm{MFR}<1.0)$ in the vast majority of patients, closely associated with an increase in duration of RS, a decrease in the severity and frequency of relapses, longer remissions after the first course of GC, a relatively slow rate of accumulation of neurological deficits (according to the EDSS disability score scale).

4. The positive dynamics of treatment led to the formation of a progressive variant of secondary progression, which has a more favorable prognostic value.

5. Patients with lack of efficacy were the most numerous and, as a rule, characterized by severe debuts, the outcome of which was protracted, accompanied by a minimal regression of the neurological deficit and a short remission after the 1st attack. As the RS progressed, there was a tendency for weight gain and lengthening of relapses, predominance of patients with high MFR value, rapid accumulation of residual neurological deficit with an increase in the rate of progression. As a result, during SSP, the course of the disease acquired an unfavorable character with the formation of a rapid rate of a steady progression.

6. As a result of the study, it was possible to prove that the effectiveness of GC therapy in the case of SPC MS is determined by the complex interaction of clinical indicators characterizing different time stages of the course of the disease, as well as a number of genetic factors that require further study.

\section{References}

[1] Shmidt, T. E., Yakhno, N. N. (2010). Rasseyannyy skleroz. Moscow: MEDpress-inform, 272.

[2] Gusev, E. I., Boyko, A. N., Stolyarov, I. D. (2009). Rasseyannyy skleroz. Moscow: Real Taym, 296.

[3] Shmidt, T. E. (2014). Lechenie i differentsialnyy diagnoz rasseyannogo skleroza i optikomielita (po materialam 29-go kongressa ECTRIMS). Nevrologicheskiy zhurnal, 19 (2), 53-62.

[4] Sadiq, S. A., Simon, E. V., Puccio, L. M. (2010). Intrathecal methotrexate treatment in multiple sclerosis. Journal of Neurology, 257 (11), 1806-1811. doi: http://doi.org/10.1007/s00415-010-5614-4

[5] Negreba, T. V. (2006). The course and prognosis of modern forms of multiple sclerosis. Ukrainskyi visnyk psykhonevrolohii, 14 (1 (46)), 44-46.

[6] Negreba, T. V. (2009). Techenie i prognoz sovremennykh form rasseyannogo skleroza: Svidotg stvo pro reestratsiyu avtors'kogo prava na tvir No. 30251 vid 15.09.2009. 
[7] Voloshyna, N. P., Vasylovskyy, V. V., Negreba, T. V. et. al. (2013). Copyright certificate No. 47786 date 13.02.2013. New approaches to clinical diagnostics of various courses of multiple sclerosis and their differentiated therapy. Practical recommendations.

[8] Vasilovskiy, V. V., Negreba, T. V., Voloshina, N. P., Chernenko, M. E. (2015). Effektivnost' mitoksantrona $\mathrm{u}$ bol'nykh s progredientnymi tipami techeniya rasseyannogo skleroza. Mizhnarodniy nevb rologichniy zhurnal, 7 (77), 17-27.

[9] Voloshyna, N. P., Vasylovskyy, V. V., Chernenko, M. Ye. (2008). The use of mitoxantrone in combination with cortexin for the treatment of progressive multiple sclerosis. Mezhdunarodnyiy nevrologicheskiy zhurnal, 6 (22), 13-16.

[10] Voloshina, N. P., Negreba, T. V., Vasilovs'kiy, V. V. (2013). Novi pidkhodi do klinichnoi diage nostiki riznikh tipiv perebigu rozsiyanogo sklerozu i ikh diferentsiyovana terapiya. Praktichni rekomenf datsii. Avtors'ke pravo na naukoviy tvir No. 47786 vid 13.02.2013.

[11] Pozer, Ch. (1993). Multiple sclerosis. Zhurnal nevropatologii i psihiatrii im. S. S. Korsakova, $4,77-78$.

[12] Voloshyna, N. P., Ehorkyna, O. V., Chernenko, M. E., Vorobeva, T. H. (2010). Sovremennie pryntsypi lechenyia rasseiannoho skleroza. Therapia. Ukrainskyi medychnyi visnyk, 2 (44), 31-33.

[13] Alam, S. M., Kyriakides, T., Lawden, M., Newman, P. K. (1993). Methylprednisolone in multiple sclerosis: a comparison of oral with intravenous therapy at equivalent high dose. Journal of Neurology, Neurosurgery \& Psychiatry, 56 (11), 1219-1220. doi: http://doi.org/10.1136/jnnp.56.11.1219

[14] Vasilovskiy, V. V., Voloshina, N. P., Negreba, T. V., Levchenko, I. L., Chernenko, M. E., Tkacheva, T. N. (2014). Opyt primeneniya i effektivnost' endolyumbal'nogo vvedeniya glyukokortikoidov pri progredientnykh tipakh techeniya rasseyannogo skleroza. Ukrains'kiy nevrologichniy zhurnal, 1 (30), 68-74.

[15] Zavalishin, I. A., Peresadova, A. V. et. al. (2011). Voprosy diagnostiki i lecheniya rasseyannogo skleroza. Russkiy meditsinskiy zhurnal, 19 (7), 468-475.

[16] Tompson, A., Polman, K., Holfeld, R. (Eds.) (2001). Multiple sclerosis: Clinical aspects and controversial issues. Saint Petersburg: Politehnika, 422.

[17] Evtushenko, S. K., Derevyanko, I. N. (2006). Modern approaches to the treatment of multiple sclerosis: achievements, disappointments, hopes ( $2^{\text {nd }}$ message). Mezhdunarodnyiy nevrologicheskiy zhurnal, $2(6), 2-12$.

[18] Gusev, E. I., Zavalishin, I. A., Boyko, A. N. (2004). Multiple sclerosis and other demyelinating diseases. Moscow: Miklosh, 540.

[19] Voloshyna, N. P., Vasylovskyy, V. V., Negreba, T. V., Levchenko, I. L., Tkacheva, T. N. (2013). Clinical description of multiple sclerosis onsets in different courses, considering the current prognosis. Ukrainskyi nevrolohichnyi zhurnal, 4 (29), 7-13.

[20] Voloshyna, N. P., Nehreba, T. V., Levchenko, I. L., Tkachova, T. M., Yehorkina, O. V. (2011). Prohnostychni kryterii pry riznykh typakh perebihu rozsiianoho sklerozu. Avtorske pravo na naukovyi tvir No. 39160 vid 14.07.2011. 